\title{
Field diagnosis of paramyxoviral infection in psittacines
}

\author{
M.T. Rani' ${ }^{1}$ P.M. Deepa ${ }^{2}$, K.Vijayakumar ${ }^{3}$ Prejith $^{4}$ \\ Department of Veterinary Epidemiology and Preventive Medicine, \\ College of Veterinary and Animal Sciences, Pookode. \\ Kerala Veterinary and Animal Sciences University, Pookode, Wayanad, India
}

Citation: Rani.M.T., Deepa,P.M., Vijayakumar,K., and Prejith. 2021. Field diagnosis of paramyxoviral infection in psittacines. J. Vet. Anim. Sci.52(1):105-106.

DOI: https://doi.org/10.51966/jvas.2021.52.1.105-106

Received : 16.08 .2019

Accepted: 30.09.2019

Published: 01.01.2021

\begin{abstract}
There are about 356 species of psittacines in the world and number of people keeping parrots as pets is innumerable. In spite of the rapid growth of Indian aviculture industry, lack of proper field diagnosis of diseases in these birds is a burning issue faced by both veterinarians and owners. Paramyxoviral infection is a threat to the aviculture industry since it spreads quickly and results in high mortality. Differential diagnosis in parrots showing neurological and respiratory signs is of paramount importance. In the present study 11 parrots namely cockatiels and alexandrine parakeets showing neurological and respiratory signs were screened for the presence of paramyxoviral infection using NDV antigen lateral flow kits. All the birds tested were positive for paramyxoviral infection. These lateral flow kits can act as a good field diagnosis for paramyxoviral infection in psittacines. The present study was the first to use lateral flow kits to identify infectious causes of neurological signs in birds of Kerala.
\end{abstract}

Keywords: Paramyxoviral infection, psittacines, field diagnosis

Bird-keeping is an emerging trend of the era. Indian aviculture industry has gone a long way since the past few decades. People keep birds either as a hobby or as a source of income. But the field of avian medicine is still at the budding stage in the country. Proper diagnosis or treatment of diseases in birds is not practised by veterinarians. Of all the diseases, neurological diseases pose a grave threat to the whole aviculture industry. Neurological signs can be due to infectious and non -infectious causes. The infectious etiologies of nervous signs includes Newcastle disease by avian paramyxovirus serotype 1, avian encephalomyelitis, septicemic form of avian listeriosis, otitis interna. Out of the many diseases, paramyxoviral infection is a threat as it spreads quickly

1. MVSc Scholar

2. Assistant Professor

3. Professor and Head, Department of Veterinary Epidemiology and Preventive Medicine, CVAS, Mannuthy

4. Assistant Professor, Department of Veterinary Public Health

Copyright: (c) 2021 Rani et al. This is an open access article distributed under the terms of the Creative Commons Attribution 4.0 International License (http://creativecommons.org/licenses/by/4.0/), which permits unrestricted use, distribution, and reproduction in any medium, provided the original author and source are credited. 
in the aviary and causes high mortality. Lack of vaccinations for paramyxoviral infection in psittacines is another factor of concern. If proper diagnosis is done at an earlier stage, the whole aviary can be protected by isolating the affected birds. Most of the tests for diagnosis of paramyxoviral infection is laborious and expensive. It is high time that the veterinarian start to rely on field diagnostic kits like NDV antigen lateral flow kits for easy diagnosis of diseases in birds.

Birdsfromthreedifferentaviarieswerepresented with neurological and respiratory signs. Birds presented included seven cockatiels and four alexandrine parakeets. Faecal samples of these birds were collected and NDV antigen lateral flow kits (Bionote, Korea) were used to detect the presence of paramyxoviral infection.

Out of the 11 birds tested for presence of paramyxoviral infection using NDV antigen lateral flow kits, all were positive which was indicated by the formation of bands in test and control in the kit. That is, 100 per cent positivity was obtained in the birds that showed nervous signs. This is the first time that these kits are being used to diagnose the presence of paramyxoviral infection in psittacines in India.

The main reason for the occurrence of paramyxoviral infection in these birds can be attributed to the presence of feral pigeons that visited the aviaries to feed on the waste materials thrown in the aviary premises. According to De Sousa et al. (2010) feral pigeons are reservoirs of paramyxoviral infection and can act as source of infection to other birds. Diagnosis of paramyxoviral infection in psittacines was earlier done using RT-PCR by Peroulis and O'Riley (2004). The antigen NDV kits used in this study are 94.7 per cent sensitive and 96.4 per cent specific compared to RT-PCR. The kits have been used in Japanese quails by Al Ajeeli and Amer (2016).

In India, there are frequent reports of psittacines being presented with neurological signs. As of now, a field diagnostic test that is simple and efficient is not been used in Indian avian diagnostic protocols. The rapid antigen NDV kits used in this study are non-invasive there by presenting an easy method for the detection of viruses and can act as a field diagnostic test for detection of NDV in pigeons.

\section{References}

Al-Ajeeli, K.S. and Amer, A.A. 2016. Serological study for detection of newcastle disease virus in Japanese quails in some state of Diyala province, Iraq. Diyala Agric. Sci. J. 8:13-21.

De Sousa, E., Júnior, A.B., Pinto, A.A., Machado, R.Z., Carrasco, A.D.O.T., Marciano, J.A. and Werther, K. 2010. Prevalence of Salmonellaspp. antibodies to Toxoplasma gondii, and Newcastle disease virus in feral pigeons (Columba livia) in the city of Jaboticabal, Brazil. J. Zoo Wildl. Med. 41:603-607.

Peroulis, I. and O'Riley, K. 2004. Detection of avian paramyxoviruses and influenza viruses amongst wild bird populations in Victoria. Aus. Vet. J. 82:79-82. 